\title{
La forma urbana efecto de la concentración dispersión de la población en Culiacán Rosales.
}

\author{
The urban form effect of population and housing dispersion concentration in \\ Culiacan Rosales.
}

Recibido: Mayo 2018

Aceptado: Agosto 2019

\section{Resumen}

Este trabajo es un avance de investigación en proceso. Se estudia la dispersión o concentración urbana a través de indicadores de densidad de población y vivienda construidos en el contexto de Culiacán Rosales, en un intento de explicar la forma urbana que tiene entendida como la estructura urbana que adquirió producto de las diferentes formas en que la población ha ocupado el suelo al asentarse en la ciudad partiendo de las teóricas de los usos de suelo urbano y del modelo monocéntrico, y sus derivados policentricos, definidos en la escuela de Chicago. Las distribución de las actividades económicas es también un factor importate que explica también la forma urbana de la ciudad, en este articulo no se analiza.

$\mathrm{El}$ articulo concluye con algunas reflexiones, resultado de las teorías y los indicadores de densidad de población y vivienda analizados, en la búsqueda de las posibles causas que expliquen la actual forma urbana que adquirió la ciudad de Culiacán Rosales.

\section{Palabras Clave:}

Forma urbana; Concentración dispersión; Densidad de población y vivienda; Culiacán Rosales.
Jorge Javier Acosta Rendón ${ }^{1}$

\begin{abstract}
This work is a research in progress. The urban dispersion or concentration is studied through population and housing density indicators constructed in the context of Culiacán Rosales, in an attempt to explain the urban form that is understood as the urban structure that acquired product of the different ways in which the population has occupied the land by settling in the city based on theories of urban land uses and the monocentric model, and its polycentric derivatives, defined at the Chicago school. The distribution of economic activities is also an important factor that also explains the urban form of the city, this article is not analyzed.

The article concludes with some reflections, the result of the theories and indicators of population and housing density analyzed, in the search for possible causes that explain the current urban form that the city of Culiacán Rosales acquired.
\end{abstract}

\section{Keywords:}

Urban form; Dispersion concentration; population and housing density; Culiacan Rosales.

\footnotetext{
${ }^{1}$ Nacionalidad: mexicano; adscripción: Universidad Autónoma de Sinaloa, Facultad de Arquitectura; Doctor en Ciudad, Territorio y Sustentabilidad. Profesor del Núcleo Académico Básico del posgrado de la Facultad de Arquitectura de la Universidad Autónoma de Sinaloa. Miembro del Sistema Nacional de Investigadores nivel I; correo electrónico: jacoren5573@uas.edu.mx
} 


\section{Introducción.}

El el presente articulo ${ }^{2}$ se analizan los conceptos duales concentración dispersión utilizando la distribución espacial de las densidades habitacionales de la población residente de Culiacán Rosales ${ }^{3}$. Se espera con este análisis tener una visión clara en cuanto a la concentración o dispersión de la población y la relación que estas dinámicas variables urbanas tienen con la forma urbana de esta ciudad a partir de las formas de ocupación del suelo, entendiendo la concentración como la forma en que un grupo de población ocupa el espacio urbano, considerando que cuanto más pequeña sea el área que un grupo ocupa mayor es su concentración; en tanto que la dispersión, proceso inverso menos frecuente por la creciente ocupación del espacio urbano, consiste en la progresiva salida de población de algunos sectores o zonas de la ciudad hacia la periferia u otras ciudades, para finalizar teniendo elementos de juicio que determinen si Culiacán Rosales es una ciudad concentrada o dispersa.

\section{Agotamiento de los modelos teóricos.}

Desde la ultima década del siglo veinte han sido fuertemente cuestionadas las teorias que tratan de explicar los usos del suelo urbano basadas en los modelos monocentricos ${ }^{4}$, derivados de la escuela de ecología humana de Chicago, desde el modelo de los círculos concéntricos de Burguess desarrollada en 1925, el modelo sectorial de Hoyt desarrollado en 1939, y el modelo de núcleos múltiples de harris y ullman desarrollado en 1945; debido a su incapacidad para explicar de manera general las actuales formas de organización y ocupación de los espacios urbanos y la localización de las actividades económicas basadas en los usos del suelo, ya que explican solo en un momento determinado el crecimiento de algunas grandes ciudades (Pinto, 2007, 85).

El modelo de Burguess concebia la ciudad como un sistema de zonas monofuncinales de uso del suelo organizadas en anillos y sectores alrededor de un distrito central de negocios especializado (CBD) en usos comerciales y administrativos. Por su parte Hoyt estableció 8 factores relacionados con las condiciones de la vivienda y el entorno residencial que trataban de explicar la localización y desarrollo residencial por medio de tres proposiciones: 1) Las personas de mayor nivel socioeconómico prefieren localizarse en áreas en las cuales tengan acceso al lujo y/o servicios; 2) Los residentes de mayor nivel socioeconómico utilizan su automóvil para desplazarse entre la casa y el trabajo, por lo tanto pueden ubicarse lejos de las áreas industriales pero cerca de los principales ejes viales; y 3) Los uso del suelo similares se agrupan para crear y/o potenciar el desarrollo del "sector". Harris y Ullman en su modelo de núcleos múltiples encontraron que las ciudades estaban desarrollando áreas de tamaño significativo que no se ligaban directamente con el CBD principal sino con centros más pequeños ubicados hacia la periferia. Encontraron además que existían ciudades que no se desarrollaban a partir de un CBD central sino que éste se forma dada la integración progresiva de núcleos separados, que posteriormente se definieron como subcentros, los cuales en tanto se desarrollan se especializan y diferencian (Pinto, 2007, 174).

La perdida de vigencia de los modelos tradicionales propició la emergencia de nuevos enfoques teóricos que pretenden describir y explicar desde diferentes disciplinas la forma como se organizan y funcionan ahora los espacios urbanos de las ciudades, como la teoría de los sistemas complejos y la de subcentros. En este contexto algunos estudiosos de la ciudad consideran que el capital económico global puede explicar la creación de estos subcentros que gradualmente sustituyen al centro tradicional $\mathrm{y}$ han propiciado el estudio de la ciudad

\footnotetext{
${ }^{2}$ Este trabajo es un producto del proyecto de investigación científica aplicada 0247832 "análisis georeferenciado de la división social del espacio en Culiacán Rosales, Sinaloa" aprobado por el Consejo Nacional de Ciencia y Tecnología en la convocatoria Proyectos de Desarrollo Científico para atender Problemas Nacionales de 2014.

${ }^{3}$ Culiacán de Rosales es una ciudad media, capital del Estado de Sinaloa, localizada al noroeste de la república mexicana entre las coordenadas $106^{\circ} 56^{\prime \prime} 50^{\prime \prime}$ y $107^{\circ} 50^{\prime \prime} 15^{\prime \prime}$ de longitud oeste y $24^{\circ} 02^{\prime \prime} 10^{\prime \prime}$ y $25^{\circ} 14^{\prime \prime} 56^{\prime \prime}$ de latitud norte. Tiene una altitud promedio de $53 \mathrm{msnm}$ (Enciclopedia de los Muncipios de México. Sinaloa. http://www.inafed.gob.mx/)

${ }^{4}$ En el modelo mononcéntrico predominante desde los años sesenta la ciudad es circular y tiene dos grandes tipos de usos del suelo, el de empleos y el residencial, donde los empleos están en un círculo central. Dada una restricción presupuestal, las familias gastan más en suelo cerca del centro si prefieren ahorrar en transporte, o gastan más en transporte si ahorran en suelo con una localización más alejada del centro (Alegría, 2009, 57).
} 
considerando la existencia de múltiples centros (policéntrica). Este fenómeno incide también en la forma urbana más evidente en las grandes ciudades que tienen buena infraestructura tecnológica para la información y comunicación (TIC) que los grandes capitales aprovechan para desarrollar magnos centros comerciales, consorcios y corporativos alejados de los centros tradicionales pero cerca de las viviendas de los empleados y de las principales vías de acceso. Sin embargo la forma urbana de las grandes ciudades es la que más se aleja de los prototipos basados en el modelo monocéntrico pues en estas la mayor parte de las actividades económicas ya no se realizan en su centro tradicional sino mas bien se observa una dispersión generalizada que se sustenta en la accesibilidad que permite el automóvil y la construcción de grandes obras de infraestura vial que genera ventajas de localización (Alegría, 2009, 64).

\section{Metodología.}

En este análisis se utilizó la carta vectorial de 2015 del INEGI de la ciudad de Culiacán Rosales para dividir la mancha urbana en cuatro circulos concéntricos, llamados contornos, cuyo centro geométrico coincide con la "zona centro" o "centro" de la ciudad. Cada circulo esta formado por las AGEB censales que encierra. El primer circulo corresponde al "Centro", o zona centro, y tiene un radio de $1 \mathrm{~km}$, el Contorno 1 , circulo contiguo al "Centro", tiene un radio de $3 \mathrm{~km}$; el contorno 2 tiene un radio de $5 \mathrm{~km}$ y el contono 3, circulo más periférico, tiene un radio de $7.5 \mathrm{~km}$. La finalidad de esta divisón geográfica de la estructura urbana de Culiacán Rosales es explorar la forma urbana a través del análisis de la distribución espacial de las densidades habitacionales utilizando estos contornos. Se define la zona más central como la zona con mayor grado de centralidad porque en ella se realiza la mayoría de las actividades terciarias. En este caso coincide con el centro geográfico de esta ciudad.

La tendencia estadística de la distribución de las densidades de población y vivienda en el espacio urbano de Culiacán Rosales se determinan con una regresión semiparametrica donde la variable independiente explica el efecto de la distancia lineal desde la zona central hasta la periferia y la variable dependiente determina el porcentaje acumulado de la población residente, y de las viviendas particulares habitadas, contenidas en las AGEB censales de cada contorno. La regresión se realizo con las densidades de población y vivienda sumando los porcentaje existentes en las AGEB censales contenidas en cada contorno de tal modo que los porcentaje de la población, y la vivienda, acumulados en cada contorno se expresan como el porcentaje del total existente en la ciudad. Así los porcentajes correspondientes al contorno 3, el más alejado del centro, equivalen al 100 por ciento de la población residente y de las viviendas particulares.

Los modelos de regresión lineal para la distribución espacial de las densidades de población y vivienda son:

$$
\begin{aligned}
& \% \mathrm{Py}=6.1324 \mathrm{Dx}-0.2963 \\
& \% \mathrm{Vy}=5.9149 \mathrm{Dx}+0.601
\end{aligned}
$$

donde:

$\mathbf{\% P y}=$ Porcentaje de la población total acumulada desde la zona centro de la ciudad hasta el contono más periférico.

$\mathbf{\%} \mathbf{V y}=$ Porcentaje del total de viviendas acumulada desde la zona centro de la ciudad hasta el contono más periférico.

$\mathbf{D x}=$ Distancia lineal desde el punto más céntrico de la ciudad hasta el limite exterior de cada contorno.

Estos modelos tienen un coeficiente de determinación o bondad de ajuste $\left(\mathrm{R}^{2}\right)$ de 0.8319 equivalente al cuadrado del coeficiente de correlación de Pearson.

\section{Distribución espacial de la población}

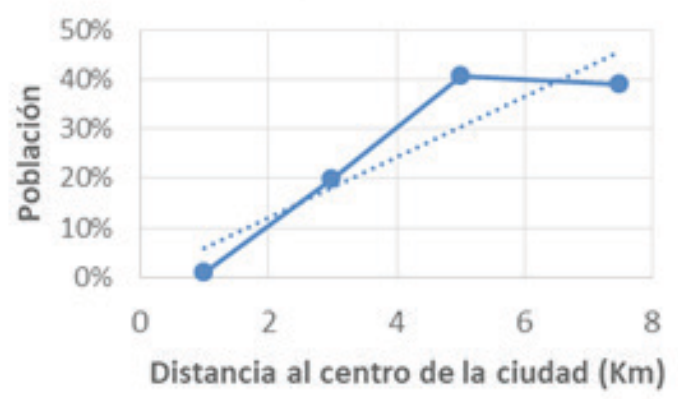




\section{Distribución espacial de la vivienda}

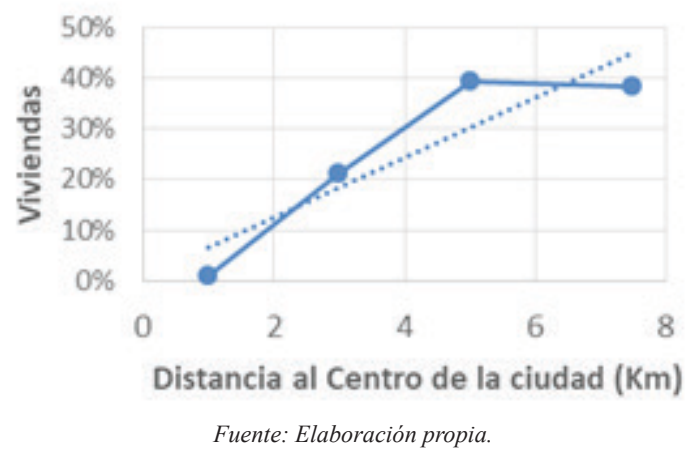

\section{Forma urbana concentrada 0 dispersa.}

La forma urbana de una ciudad puede definirse, de acuerdo con Tito Alegría $(2009,169)$, como el patrón que representa la distribución espacial de la población residente y las actividades económicas que en ella se realizan conceptualizada y definida por diferentes teorías entre ellas los usos del suelo urbano. Explica que la definición más utilizada para explicar los usos del suelo esta basada en el modelo moncentrico y sus derivados policéntricos emanados de la escuela de Chicago, y sus posteriores derivados, sin embargo como se dijo estos, estos han mostrado su agotamiento teórico para determinar el crecimiento de la mayoría de la ciudades, sobre todo las consideradas grandes.

De acuerdo con Emilio Pradilla Cobos $(2011,5)$ en las ultimas décadas las ciudades han cambiado hacia una forma difusa, caotica, dispersa, expandida, estallada, derramada, archipiélago, y otras denominaciones que según otros autores se popularizan en muchos textos como "caracterizaciones teóricas" de formas y etapas actuales como modelos inevitables del devenir de las ciudades en todos los paises del mundo. Además las tecnologías de la edificación, la infraestructura y la movilidad también son determinantes de la forma que adquiere la estructura urbana y el medio ambiente, según Burgess $(2011,67)$. Incluso, la emergencia de nuevos asentamientos informales (imagen 1) en la periferia de las ciudades y la modalidad de encerramiento de los asentamientos de clase media y alta al interior de la ciudad, se incluyen tambien como un importante factor que influye en la configuración de la forma urbana de la ciudad.
Figura 1. Asentamiento informal y fraccionamiento cerrado en Culiacán Rosales.
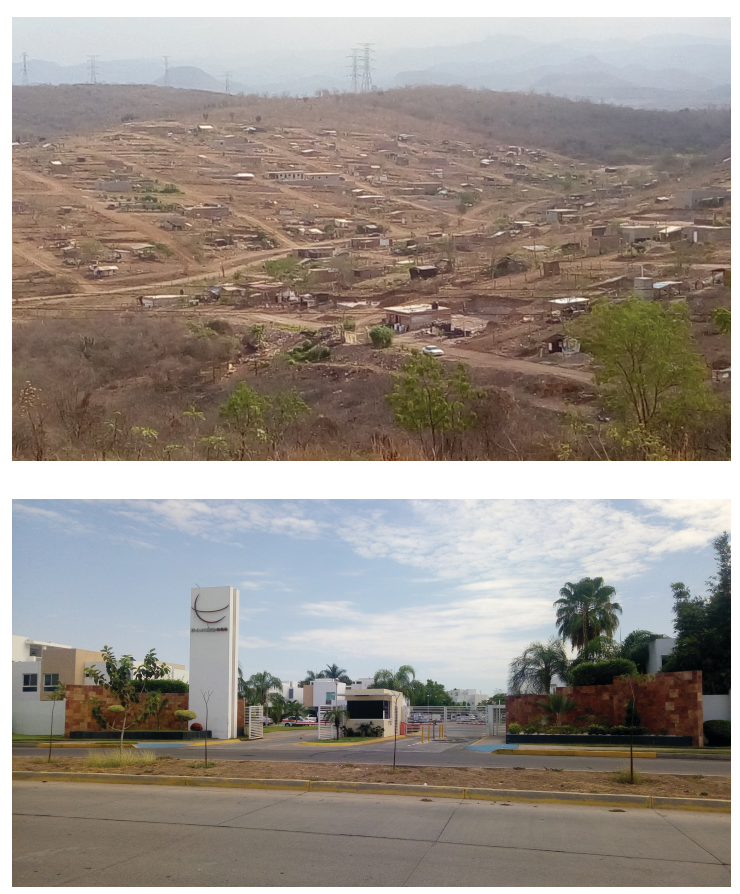

Fuente: Propia. 2017.

Según López Rangel $(2011,229)$ la forma urbana de la ciudad esta vinculada de manera compleja con los "contenidos", es la configuración geométrica regular o irregular de una "mancha urbana" que se asienta y desparrama en el territorio tanto a nivel de sus delineados externos como a sus densidades externas. Expone que en la extensión de la mancha urbana con su estructura de vialidades, espacios abiertos y cerrados, publicos y privados, se observan movimientos complejos de "ida y de regreso" con diferentes velocidades, direcciones y retroacciones, segun el punto de referencia, que generan nuevas centralidades y "lineas de desarrollo formal" que se expanden en diferentes direcciones que junto a las densidades habitacionales cuentan en la forma urbana de la ciudad.

López Rangel explica también que la organización formal de la ciudad se complica si consideramos que en la ciudad conviven de manera constante y simultánea fenómenos relacionados con aspectos económicos, sociales, ambientales, demográficos, culturales, educativos, delicuenciales, etc., que dividen, fragmentan y segregan los espacios urbanos, lo que intuye la complejidad para analizar el funcionamiento de toda la estructura espacial urbana, que en realidad 
conforma el sistema ciudad, lo que hace necesario utilizar modelos matematicos ${ }^{5}$ para su análisis desde la perspectiva cuantitativa. Concluye que la formación de nuevas colonias, fraccionamientos, conjuntos, pueblos tradicionales históricos $\mathrm{y}$ asentamientos habitacionales, con sus diferentes modalidades, así como la conurbación de municipios en las ciudades grandes, han influido en la forma urbana de las ciudades mexicanas. (Lopez Rangel, 2011, 230).

En este contexto lo que sorprendio a Emilio Duhau al observar enormes conurbaciones como la ciudad de México es más bien lo contrario, es decir, una persistente tendencia a que los procesos de poblamiento tiendan a generar una aglomeración compacta. Lo que ocurre, explica, es que cuando se producen esos "huecos" o espacio urbanizado no utilizado (imagen 2), se "rellenan" con el tiempo (Duhau, 2008, 164).

Según el enfoque socioespacial, analizado por Janoshka (2004), las etapas del desarrollo urbano en las ciudades están relacionados con los cambios en la economía política de la sociedad. Aun se discute la forma exponencial en que ha ocurrido el crecimiento urbano sin respetar los patrones naturales que le han dado forma a la estructura de las ciudades en búsqueda de satisfacer las necesidades espaciales de una sociedad de consumo en crecimiento. En este contexto la ciudad es un espacio físico con vida propia que nace, crece y se desarrolla a consecuencia de factores económicos, sociales, culturales diferentes, tal como lo apunta Martha Schteingard $(2010,348)$ "una ciudad es producto de la historia y los fenómenos que en ella ocurren afectan a la ciudad como una unidad".

Figura 2. Huecos urbanos en Culiacán Rosales.

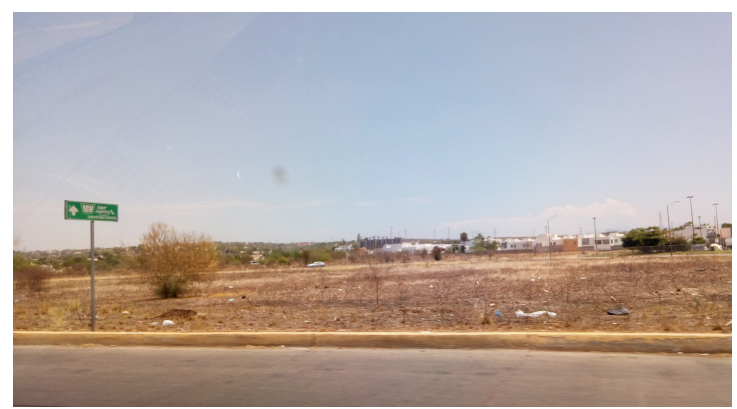

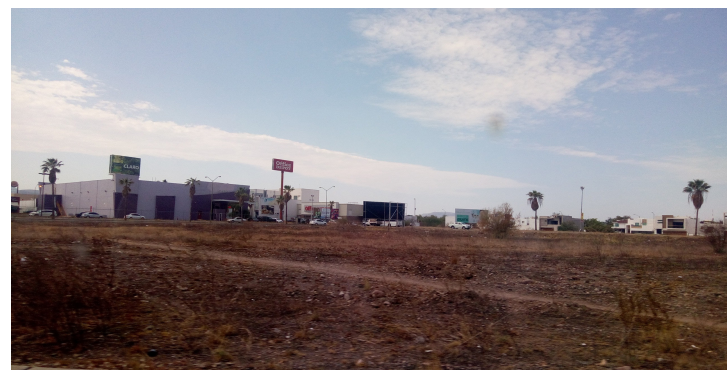

Fuente: Propia. 2017.

Sin embargo el crecimiento urbano no siempre se acompaña del desarrollo. El crecimiento de la ciudad alude a cuestiones materiales y objetivas relacionadas con el aspecto económico, con el tener, como tener casa, automóvil, bienes, una embarcación; los edificios, la calle, el parque, etc., el crecimiento es entonces tangible. En tanto que el desarrollo es un proceso subjetivo que se relaciona con el ser, encierra aspectos como educación, cultura, empleo, la familia, gobierno, gobernabilidad, relaciones sociales, etc. En este aspecto donde entran los fenómenos urbanos de división social y segregación que evidencian crecimiento sin desarrollo. El desarrollo es entonces intangible (Boisier, 2010). Por tanto los procesos contemporáneos del desarrollo económico llevan a la forma urbana a estar concentrados o dispersos, de esta manera la forma de los espacios ue integran los asentamientos de la población esta relacionada con los modos de organización de la economía (Imagen 3). Lo anterior significa que los patrones espaciales importantes que definen la organización espacial de la sociedad, que a su vez definen la forma urbana, están correlacionados con aspectos específicos de las características económicas, políticas y culturales de los modos de organización de la sociedad.

Figura 3. Desarrollo comercial en Culiacán Rosales.

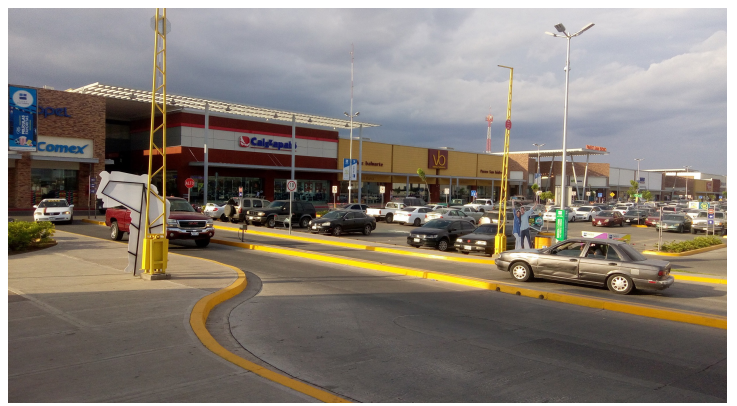

\footnotetext{
${ }^{5}$ Actualmente se disponde de diversos instrumentos cuantitativos para el análisis del territorio que utilizan metodos factoriales multivariable
} que se sustentan en la correlación entre proposiciones analíticas y sintéticas para reemplazar a la realidad mediante axiomas no evidentes. 


\section{Distribución espacial de las densidades de habitacionales.}

De acuerdo con Tito Alegría (2009, 174), la distribución espacial de las densidades de población es una de las principales características en el análisis urbano. En el modelo monocéntrico la densidad decrece desde el centro hacia la periferia. Expone que este modelo explica la distribución espacial de la densidad como resultado del comportamiento de la oferta y la demanda de vivienda al enfrentar el precio del suelo, el cual decrece desde el centro hacia la periferia de la ciudad. Así por el lado de la oferta los constructores utilizan más el factor que menos cuesta por unidad de vivienda, por ello, a medida que se acercan al centro de la ciudad utilizan más materiales de construcción y menos suelo, como resultado aumentan el número de pisos y con ello la densidad habitacional. Por el lado de la demanda las familias con ingresos bajos pueden elegir gastar más en suelo y tener menos metros cuadrados de superficie con mayor densidad si deciden asentarse cerca del centro, porque cerca del centro el valor del suelo es más alto pero se reduce el gasto del transporte, en comparación si eligieran asentarse en la periferia.

Según el Censo de Población y Vivienda de 2010 del INEGI la ciudad de Culiacán Rosales tenía 675,773 habitantes (hab) y 176,799 viviendas particulares habitadas (vph) que sumadas a las 35,533 deshabitadas y 8,812 de uso temporal hacen un total de 221,144 viviendas (Gráfico 1 y tabla 1)) distribuidas en un territorio urbanizado que cubría 12,584.50 hectáreas (ha). Por tanto en ese momento las densidades habitacionales promedio eran $53.7 \mathrm{hab} / \mathrm{ha}$ y $14.0 \mathrm{viv} / \mathrm{ha}$. Estas bajas densidades indican que la ciudad ha tenido un crecimiento expansivo con un alto consumo de suelo lo cual ha incidido de manera importante en la actual forma urbana que tiene esta ciudad.

En este sentido el uso habitacional del suelo, generador de la forma urbana de Culiacan Rosales creemos que tiene explicaciones similares a las que encontró Tito Alegria $(2009,175)$ en su análisis de la Metropolis Transfronteriza Tijuana, México - San Diego, Estados Unidos. De acuerdo con su análisis una primera apreciación tiene que ver con que en que las grandes ciudades la distribución de los precios del suelo no es estrictamente decreciente desde el centro debido a que hay subcentros ${ }^{6}$ terciarios que elevan el precio del suelo lejos del centro principal de la ciudad. Si la densidad de población sigue a los precios del suelo, explica, la distribución de esa densidad no será claramente decreciente desde el centro de la ciudad. Explicación que se apega más al enfoque del modelo policéntrico.

Gráfico 1. Dinámica demográfica y habitacional de Culiacán Rosales, Sinaloa.

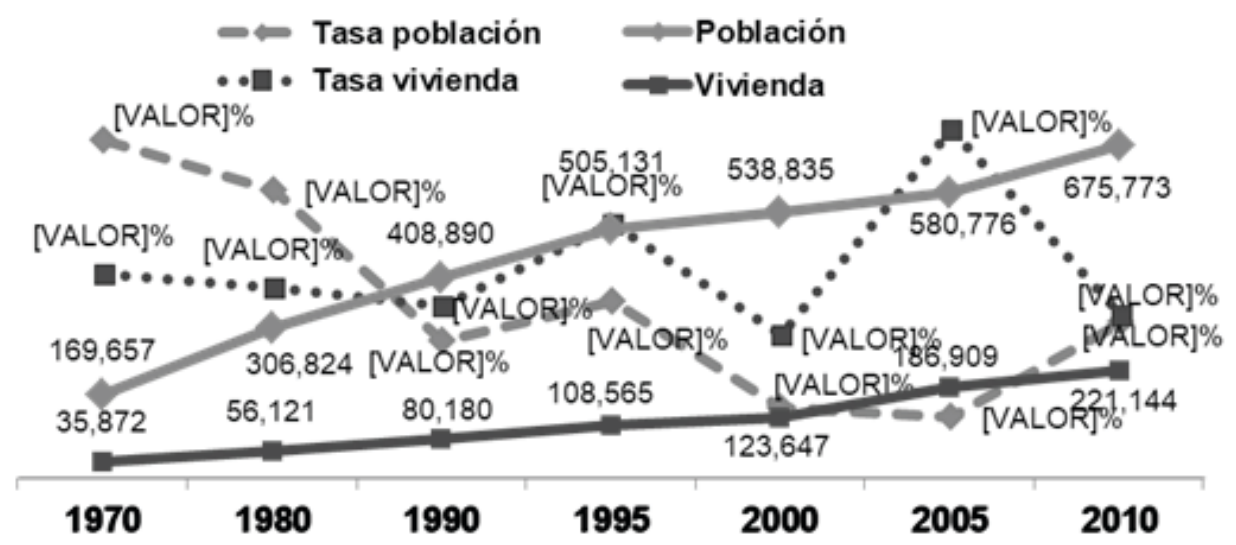

Fuente: Elaboración propia con información del INEGI 2010.

\footnotetext{
${ }^{6}$ Las debilidades del modelo monocéntrico dieron cabida al surguimiento de modelos alternativos de usos del suelo entre ellos los modelos policéntricos. Desde la visión neoclásica, estos modelos trataron de extender para dos o más centros (policentrismo) la lógica del balance entre renta del suelo y costo de transporte que hacen los agentes cuando deciden su localización intraurbana La teoría del modelo policentrico explica el funcionamiento de la ciudad considerando la emergencia actual de subcentros (endógenos o exógenos) que están sustituyendo al centro original.
} 
Tabla 1. Distribución espacial de la población y la vivienda.

\begin{tabular}{|c|c|c|c|c|c|c|c|c|c|}
\hline \multirow{2}{*}{ CONTORNOS } & \multicolumn{3}{|c|}{ POBLACIÓN } & \multicolumn{3}{|c|}{ VIVIENDA } & \multirow{2}{*}{ Hab/Viv } & \multirow{2}{*}{ Área (Ha) } & \multirow{2}{*}{$\begin{array}{l}\text { Distancia al } \\
\text { centro }(\mathrm{Km})\end{array}$} \\
\hline & $\mathrm{Hab}$ & $\%$ & $\mathrm{Hab} / \mathrm{Ha}$ & Viv & $\%$ & Viv/Ha & & & \\
\hline CENTRAL & 5,627 & 0.8 & 25.0 & 1,892 & 1.1 & 8.3 & 3.0 & 225.1 & 1 \\
\hline CONTORNO 1 & 133,022 & 19.7 & 41.2 & 37,330 & 21.1 & 11.5 & 3.6 & $3,231.7$ & 3 \\
\hline CONTORNO 2 & 273,855 & 40.5 & 65.6 & 69,638 & 39.4 & 16.7 & 3.9 & $4,175.7$ & 5 \\
\hline CONTORNO 3 & 263,269 & 39.0 & 53.2 & 67,939 & 38.4 & 13.7 & 3.9 & $4,952.0$ & 7.5 \\
\hline TOTAL & 675,773 & 100.0 & 53.7 & 176,799 & 100.0 & 14.0 & 3.82 & $12,584.5$ & \\
\hline
\end{tabular}

Una situación similar la encontramos en la ciudad de Culiacán Rosales donde las densidades crecen desde el centro a la periferia, contrario a lo que establece el modelo monocentrico, sin embargo este crecimiento es diferenciado pues existen zonas dispersas por la ciudad que concetran actividades complementarias que pueden ser subcentros en formación, escenario que estamos en proceso de estudio aplicando la regresión paramétria donde la variable dependiente explica el valor del suelo y la independiente la distancia al centro principal. En una segunda apreciación Alegría (Op.Cit.) expone que un supuesto implícito en el enfoque monocéntrico es que el mercado de la vivienda debe funcionar de manera perfecta para ajustar oferta y demanda, sin embargo, se sabe que estos tienen varias imperfecciones. Una de ellas es que, en todo el país, más de la mitad de la población urbana tuvo acceso a una vivienda fuera del mercado formal ${ }^{7}$ mediante el proceso de invasión de suelo urbano. Esto quiere decir que la densidad de población de al menos la mitad del territorio urbano de las ciudades mexicanas, en la cuales se incluye Culiacán Rosales, no se constituyó debido al precio del suelo regulado por el mercado formal sino por procesos irregulares relacionados con la invasión de tierras en las periferias urbanas. Además, la vivienda se considera patrimonio familiar cuyo desarrollo es progresivo y se extiende a lo largo de varios años, incluso décadas, en los cuales cambia la estrucutra familiar y con ello cambia también la densidad de población. Coincidimos también con la tercera apreciación de Alegría (Op.Cit.) donde expone que las actividades terciarias compiten por suelo accesible imponiendose en una localización central al ofrecer más capital por metro cuadrado donde la vivienda no puede competir igual por lo que es expulsada al cambiar el uso del suelo con lo que disminuye la cantidad de personas que residen en el centro y con ello la densidad habitacional.

En la tabla 1 anterior se muestra que en la zona centro de Culiacán Rosales las densidades de población y de vivienda son más bajas que en los contornos más periféricos. Se puede ver que estás van desde $25 \mathrm{hab} / \mathrm{ha}$ y $8.3 \mathrm{viv} / \mathrm{ha}$ en la zona centro hasta $65.6 \mathrm{hab} / \mathrm{ha}$ y $16.7 \mathrm{viv} / \mathrm{ha}$ en los contornos más alejados del centro. Sin embargo, en el contorno 3, el más periférico, las densidades son más bajas que en el contorno 2 menos periférico, debido a que en el cotorno 3 hay una gran cantidad vacíos urbanos, lotes de suelo urbanizado que permanecen ociosos en un claro proceso de especulación que el capital esta "engordando" para futuros desarrollos inmobiliarios. Por tanto, y siguiendo este enfoque, la distribución espacial de las densidades habitacionales que se presenta en Culiacán Rosales (mapa 1) no se puede explicar con el modelo monocéntrico pues en esta ciudad, como ya se explicó, la densidad habitacional es más alta en la periferia contrario a lo que explica el modelo moncéntrico.

\footnotetext{
${ }^{7}$ El mercado formal de la vivienda en México es que oferta vivienda construida por desarrolladores inmobiliarios financiada por organismos nacionales como el INFONAVIT, principalmente.
} 


\section{Reflexiones finales.}

Considerando que Culiacán Rosales es una ciudad media en la que su centro tradicional aun concentra la mayor cantidad de los empleos y las actividades económicas dominantes, desde el punto de vista económico su forma urbana puede ser explicada por el modelo monocéntrico dado que no muestra evidencias contundentes de que en su territorio se estén formando subcentros sino mas bien se observa una dispersión generalizada de actividades terciarias sustentadas en la accesibilidad que permite el automóvil y la construcción de infraestura vial que genera ventajas de localización. Sin embargo, dispersos también por diferentes zonas de la ciudad, empiezan a emerger concentraciones de actividades terciarias, tipo plazas comerciales con tiendas de marcas globales, que se hace necesario estudiarlas con más herramientas para el analisis urbano donde se consideren otras variables como el valor del suelo, el ingreso de los hogares, y otras variables que reflejen las condiciones del entorno, que ayuden a determinar si estos ambientes emergentes están formando subcentros y pueden tener explicaciones basadas en el modelo policentrico, y si además estos escenarios estan influyendo en la cambiante forma urbana de esta ciudad.
Por otra parte desde el punto de vista sociológico la forma urbana y los usos del suelo que definen el funcionamiento de la ciudad de Culiacán Rosales no se acomodan para que puedan ser explicados por el modelo monocéntrico pues la densidad habitacional no decrece desde el centro hacia la periferia como lo establece este modelo, sino por el contrario en este caso la densidad es más alta en la periferia, situación explicada de mejor manera por la definición de subcentros terciarios fuera del centro tradicional que sustentan al modelo policéntrico.

Otra caractrística de Culiacán Rosales que incide en la forma urbana que actualmente tiene consiste en que dispersos por toda la ciudad, aunque en mayor concentración en los contornos 2 y 3 periféricos, existe una gran cantidad de suelo urbanizado ocioso que generan grandes vacíos urbanos formados a causa de la expansión generalizada de asentamientos que se localizaron en la periferia modificando los limites urbanos de la ciudad en un proceso de especulación del capital inmobiliario, que de acuerdo con Emilio Duhau (op. cit.) son "huecos" que se "rellenan" con el tiempo después que se han revalorizado por las acciones urbanas realizadas por el municipio encauzando la ciudad hacia una forma urbana compacta. $\mathbf{G}$

Mapa 1. Distribución espacial de las densidades habitacionales en Culiacán Rosales, Sinaloa.

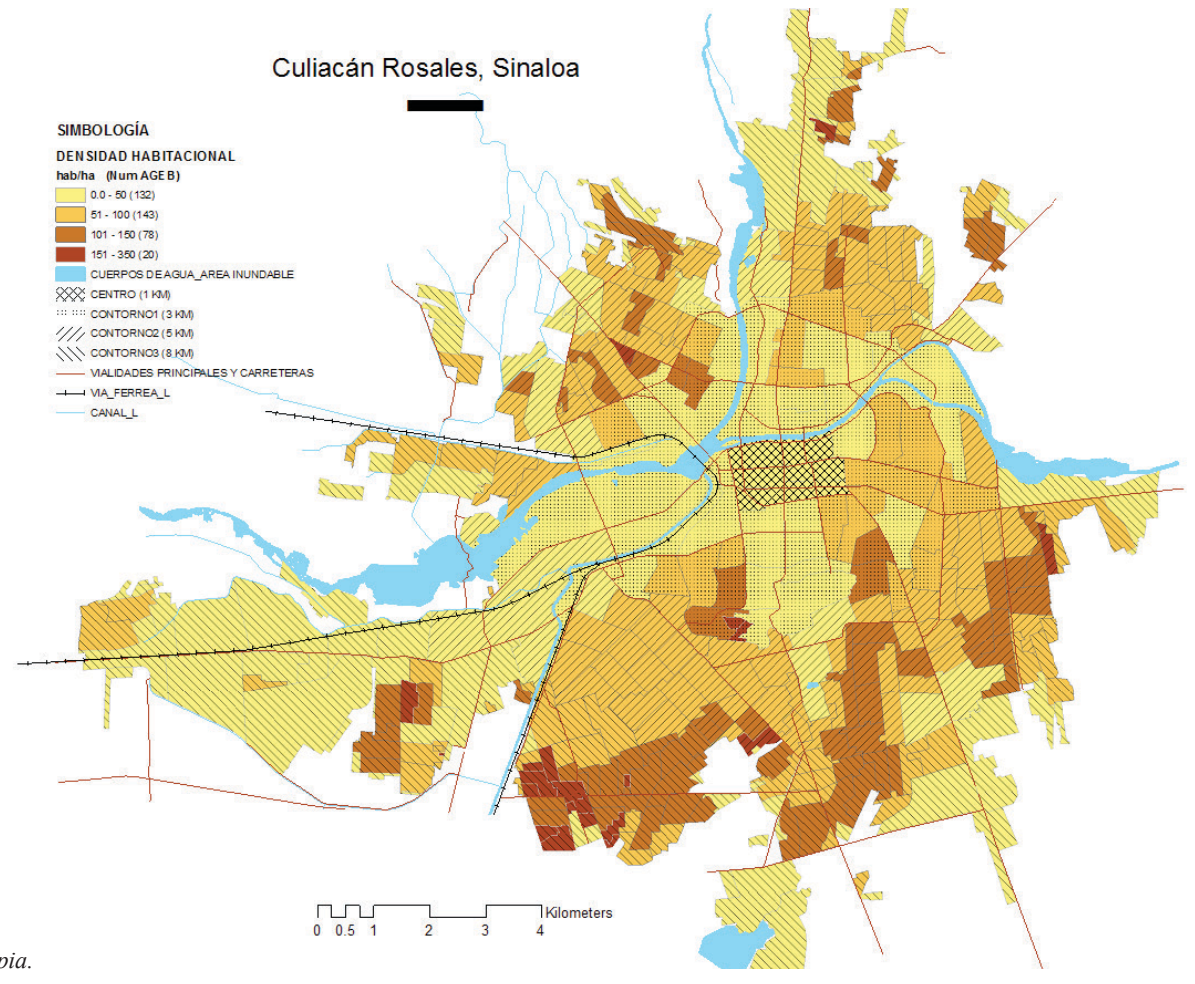




\section{Bibliografía.}

Alegría, Tito (2009). Metrópolis transfronteriza: Revisión de la hipótesis y evidencias de Tijuana, México y San Diego, Estados Unidos. $1^{\mathrm{a}}$ edición. Tijuana. El Colegio de la Frontera Norte; México, D.F. Miguel Ángel Porrúa.

Boisier, Sergio, 2010. Descodificando el desarrollo del siglo xxi: subjetividad, complejidad, sinapsis, sinergía, recursividad, liderazgo, y anclaje territorial. Semestre Económico, vol. 13, núm. 27, julio-diciembre, 2010, pp. 11-37 Universidad de Medellín. Medellín, Colombia

Burgess, Rod (2011). Determinismo tecnológico y fragmentación urbana: un análisis crítica, en Ciudades Compactas, dispersas y fragmentadas, Emilio Pradilla Cobos, Compilador. Universidad Autónoma Metropolitana. Miguel Ángel Porrúa. México.

Duhau, Emilio y Giglia, Ángela (2004). Conflictos por el espacio $y$ orden urbano. Estudios demográficos y urbanos, mayo-agosto 2004, número 056. El colegio de México, A.C., D.F., México.

Duhau, Emilio (2006). Organización del espacio urbano, segregación y espacio público; en CIUDADES 66, abril-junio de 2005, RNIU, Puebla, México.

(2008). Los nuevos productores del espacio habitable; en ciudades 79, julioseptiembre de 2008, RNIU, Puebla, México.

Los Municipios de Sinaloa (1988). Colección: Enciclopedia de los Municipios de México. Secretaría de Gobernación y Gobierno del Estado de Sinaloa. http://www.inafed.gob. $\mathrm{mx} /$.

López Rangel, Rafael (2011). De la Ciudad Compacta a la Ciudad Fragmentada: El caso de la Ciudad de México. En el Universo de las nuevas preocupaciones sobre la ciudad; del sueño de un orden a la pesadilla del caos, en Ciudades Compactas, dispersas y fragmentadas, Emilio Pradilla Cobos, Compilador. Universidad Autónoma Metropolitana. Miguel Ángel Porrúa. México.

Pinto Verdugo, Mónica (2007). Los modelos explicativos de la estructura urbana. Una visión crítica de su desarrollo al interior de la consolidación de la racionalidad moderna (no editado). Memoria para optar al título de geógrafo. Facultad de Arquitectura y Urbanismo. Escuela de Geografía. Universidad de Chile. Chile.
Pradilla Cobos, Emilio (2011). Ciudades Compactas, dispersas, fragmentadas. UAM. Miguel Ángel Porrúa. México.

Schteingart, Martha (2010). La división social del espacio en las ciudades. Revista Herramienta $\mathrm{N}^{\circ}$ 48. SociologíaCiudad y territorio. Octubre de 2011 - Año XV. 